\title{
Evolution@home: Global computing quantifies evolution due to Muller's ratchet
} Laurence Loewe*

\author{
Address: Institute of Evolutionary Biology, School of Biological Sciences, University of Edinburgh, Ashworth Laboratories, Kings Buildings, West \\ Mains Road, Edinburgh EH9 3JT, Scotland, UK. \\ Email: Laurence Loewe* - Laurence.Loewe@evolutionary-research.net \\ * Corresponding author
}

from BioSysBio: Bioinformatics and Systems Biology Conference

Edinburgh, UK, I4-15 July 2005

Published: 2I September 2005

BMC Bioinformatics 2005, 6(Suppl 3):PI8

The importance of evolutionary processes in shaping sequence patterns in genomes, as well as origin and extinction of species is well established. As the last century of research has shown, progress in these areas heavily depends on our ability to quantify verbal arguments in order to rank the significance of various ongoing processes. Most of the work in this area traditionally employs analytical mathematics, usually applying a considerable number of simplifying assumptions to become tractable. The last two decades have seen the rise of computer simulations that allow quantification for some previously intractable problems. However, solid simulation work often needs to explore a wide range of parameter space and needs enough independent repeats to allow rigorous quantification in the case of stochastic simulations. This is where problems typically arise: The conceptually high accuracy of simulated models is frequently lost due to simplifying assumptions needed to satisfy constraints in computational complexity.

Here I demonstrate the use of global computing to help quantify evolutionary models, where no dedicated supercomputer is available. To this end I had started evolution@home, the first global computing system for evolutionary biology http://www.evolutionaryresearch.net/. Its first simulator quantifies Muller's ratchet in asexual genetic systems like mtDNA. Muller's ratchet may compromise long-term fitness due to accumulation of slightly deleterious mutations, but its speed is notoriously difficult to quantify for intermediate selection coefficients. Using evolution@home I built a database that contains $>70$ years of CPU time from more than 300 participants. Stored results allow easy quantification of the threat from Muller's ratchet. Thorough analyses provide yet another reason, why anthropogenic increase of mutation rates should be minimized and suggest that quantification of rare potential recombination in mitochondria may be worth the effort. 